\title{
LAS CUENCAS EXPERIMENTALES DE ARNÁS Y SAN SALVADOR EN EL PIRINEO CENTRAL ESPAÑOL: ESCORRENTÍA Y TRANSPORTE DE SEDIMENTO
}

\author{
J. M. GARCÍA RUIZ \\ C. MARTÍ BONO \\ J. ARNÁEZ VADHLLO
}

S. BEGUERÍA PORTUGUÉS ${ }^{1}$

A. LORENTE GRIMA ${ }^{1}$

M. SEEGER ${ }^{1}$

(1) Instituto Pirenaico de Ecología, CSIC, Campus de Aula Dei, Apartado 202, 50080-Zaragoza.

C. electrónico: humberto@ipe.csic.es

(2) Area de Geografía Física (DCHS). Edif. L. Vives. Universidad de La Rioja, 26004-Logroño.

C. electrónico: jose.arnaez@dchs.unirioja.es

RESUMEN. Las cuencas experimentales de Arnás y San Salvador, localizadas en la cuenca alta del río Aragón, han sido equipadas con estación meteorológica completa, flume e instrumentos para la medición continua del caudal y del sedimentos transportado (en solución y en suspensión). Una trampa de sedimento a la entrada del flume permite controlar periódicamente la carga de fondo. Los resultados obtenidos confirman la existencia de grandes diferencias en la respuesta hidrológica y el transporte de sedimento entre la cuenca forestal (San Salvador) y la cuenca de campos abandonados (Arnás). La primera muestra una respuesta mucho más moderada debido básicamente a los procesos de interceptación y a la mayor profundidad de los suelos. Las relaciones entre precipitación y pico de caudal, así como entre este último y la concentración de sedimento en suspensión son muy bajas, aumentando notablemente si se contemplan por separado las crecidas de estación fría. La escorrentía se genera sobre todo por procesos de saturación. Las fuentes de sedimento se localizan en el cauce y sus proximidades.

ABSTRACT. The experimental catchments of Arnás and San Salvador, located in the Upper Aragón River Basin, have been monitored with a weatber station, a flume and instruments to continuously measure the discharge and the sediment carried out (as solutes and suspended sediment). A sediment trap at the flume allows to weight periodically the bedload. The results obtained confirm the existence of great bydrological and geomorpbological differences between the forest covered catchment (San Salvador) and the farmland abandoned catcbment (Arnás). The former shows a much more moderate response due basically to the process of interception and to a greater soil depth. Relationships between precipitation and peak flow, as well as between this latter and suspended sediment concentration are very low, increasing notoriously if cold season floods are considered isolately. Runoff generation is mainly due to saturation processes. Finally, sediment sources are located in the channel and its closeness. 
Palabras clave: Cuencas experimentales, Escorrentía, Crecidas, Transporte de sedimento, Pirineo Central español.

Key words: Experimental catchments, Runoff, Floods, Sediment transport, Central Spanish Pyrenees.

\section{Introducción}

Las relaciones complejas que se establecen entre generación de escorrentía y transporte de sedimentos, por un lado, y factores tales como la topografia, la cubierta vegetal o los usos del suelo, por otro, requieren información continua y muy detallada para explicar la variabilidad espacial y temporal de procesos hidrológicos y geomorfológicos. Tales relaciones sólo pueden estudiarse con precisión en cuencas de reducido tamaño, donde la heterogeneidad interna es relativamente pequeña, y donde es posible conocer la respuesta hidrológica de diferentes sectores de la cuenca frente a eventos pluviométricos de distinta magnitud (García Ruiz \& Gallart, 1997).

La necesidad de disponer de modelos predictivos para la evaluación de los recursos hídricos, el transporte de sedimento o el comportamiento de avenidas en grandes cuencas obliga a realizar esfuerzos de validación cuya eficacia es mayor en cuencas de tamaño reducido. Tales modelos, una vez validados, podrán aplicarse progresivamente a cuencas mayores, donde es evidente que las relaciones entre factores y procesos son muy dificiles de detectar.

A mediados de 1995 el Departamento de Erosión y Usos del Suelo del Instituto Pirenaico de Ecología inició la monitorización de la cuenca experimental de Arnás, en el Valle de Borau, afluente por la derecha del río Aragón. En 1998 se equipó una segunda cuenca, muy próxima a la anterior, llamada de San Salvador, en el Valle de Aísa, también afluente del río Aragón. Las razones de esta iniciativa son sencillas de explicar: Desde 1991 venía funcionando, a cargo del mismo Departamento, la Estación Experimental Valle de Aísa, donde se obtenía información continua sobre escorrentía y transporte de sedimento bajo diferentes usos del suelo, a partir de parcelas experimentales de $30 \mathrm{~m}^{2}$ de superficie (García Ruiz et al., 1996a, 1996b). Una vez identificados algunos de los problemas hidromorfológicos más importantes, se hacía necesario no sólo cambiar de escala, sino también realizar una aproximación más integradora al problema, y sin las limitaciones que presentan los resultados obtenidos en parcelas experimentales. La monitorización de cuencas permitía un salto cualitativo y cuantitativo en la obtención de información e incrementaba las posibilidades de conocer el funcionamiento interno de sistemas hidrológicos, jerarquizar los factores que explican su dinámica, elaborar balances de agua y sedimento y estudiar los efectos de los cambios de uso del suelo a más largo plazo (Walling, 1991; García Ruiz \& Gallart, 1997).

En este trabajo se presentan las características fundamentales de las dos cuencas citadas, el equipamiento y los objetivos que se persiguen, así como los resultados más destacados hasta el momento. 


\section{Las cuencas experimentales de Arnás y San Salvador}

Las dos cuencas se localizan en el sector del flysch eoceno surpirenaico (Fig. 1), con delgados estratos alternantes de areniscas y margas buzando hacia el norte y pendientes situadas predominantemente entre el 20 y el $40 \%$.

La cuenca de Arnás ocupa 284 Ha y es afluente por la derecha del río Lubierre. Su altitud máxima se alcanza a $1.330 \mathrm{~m}$, y la inferior a $900 \mathrm{~m}$. El barranco de Arnás circula de manera subsecuente de oeste a este, dando lugar a un fuerte contraste topográfico entre la ladera solana, correspondiente a un abrupto frente de cuesta, y la ladera umbría, de pendiente más suave, coincidiendo con un prolongado reverso de cuesta. La Fig. 2 muestra los rasgos geomorfológicos más importantes de esta cuenca: la ladera solana presenta varias coladas de piedras de escasa longitud y desconectadas de la red fluvial, así como cicatrices de movimientos en masa más profundos, del tipo earth flows y slumps. En la ladera umbría es frecuente la presencia de antiguas cicatrices y lenguas de movimientos en masa profundos, de edad desconocida, que indudablemente son anteriores a la ocupación humana. La erosión afecta sobre todo a las vertientes convexas de la ladera solana, con una más lenta recolonización por el matorral. No obstante, sobre el terreno no se ha podido comprobar su conexión con la red fluvial, excepto en áreas próximas al cauce.

La cuenca de San Salvador ocupa $93 \mathrm{Ha}$ y es afluente por la izquierda del río Estarrún. Alcanza su altitud máxima a $1.325 \mathrm{~m}$ y la más baja a $810 \mathrm{~m}$. No presenta fuertes contrastes entre umbría y solana y no hay evidencias de erosión, excepto en el sector más bajo de la solana, donde se asientan antiguos campos de cultivo que en su mayoría no drenan hacia el punto del cauce donde está situado el aforo.

Las dos cuencas muestras condiciones climáticas similares: predominio de un clima submediterráneo de montaña con influencias atlánticas, con una precipitación media anual próxima a $1000 \mathrm{~mm}$ en la parte baja (en Esposa, $930 \mathrm{~mm}$ a $980 \mathrm{~m}$ s.n.m.) y máximos en otoño y primavera. Las tormentas estivales son relativamente frecuentes, aunque las precipitaciones más intensas tienden a presentarse en octubre y noviembre.

El suelo predominante es el kastanozem háplico, profundo y bien estructurado, con alto contenido en materia orgánica y buena permeabilidad. Sin embargo, en la solana de la cuenca de Arnás domina el regosol calcáreo, con baja capacidad de retención de agua y elevada pedregosidad (García Ruiz et al., 1999).

La mayor diferencia existente entre las dos cuencas radica en la historia de los usos del suelo y en su actual cubierta vegetal. La cuenca de Arnás ha sido completamente cultivada hasta mediados del siglo XX mediante campos en pendiente. En la actualidad la mayor parte de las antiguas parcelas se hallan cubiertas de matorral, con tendencia a bosque abierto de Pinus sylvestris y Quercus gr. faginea en la parte alta de la umbría. El matorral está constituido por una formación densa de Buxus sempervirens, Genista scorpius, Rosa gr.canina, Juniperus communis y Echynospartum horridum. Durante todo el año la cuenca se aprovecha en régimen de pastoreo extensivo con ganado ovino.

La cuenca de San Salvador está cubierta por un bosque denso de Pinus sylvestris, con bosquetes de Fagus sylvatica en las concavidades umbrias de la parte alta y Quercus gr.faginea dispersos en la parte baja. No hay evidencias de pastoreo reciente. 


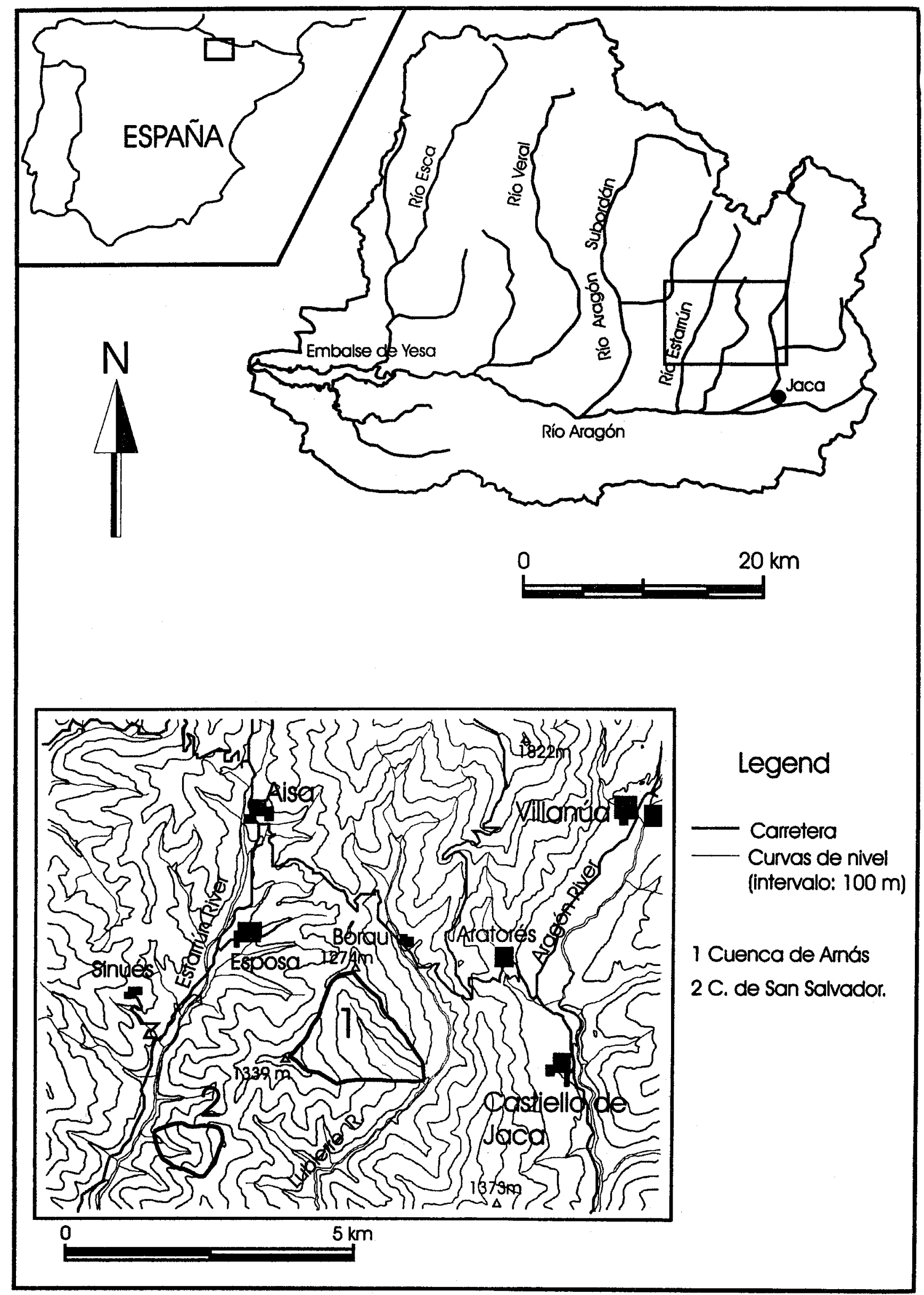

Figura 1. Localización de las cuencas de Arnás y San Salvador 


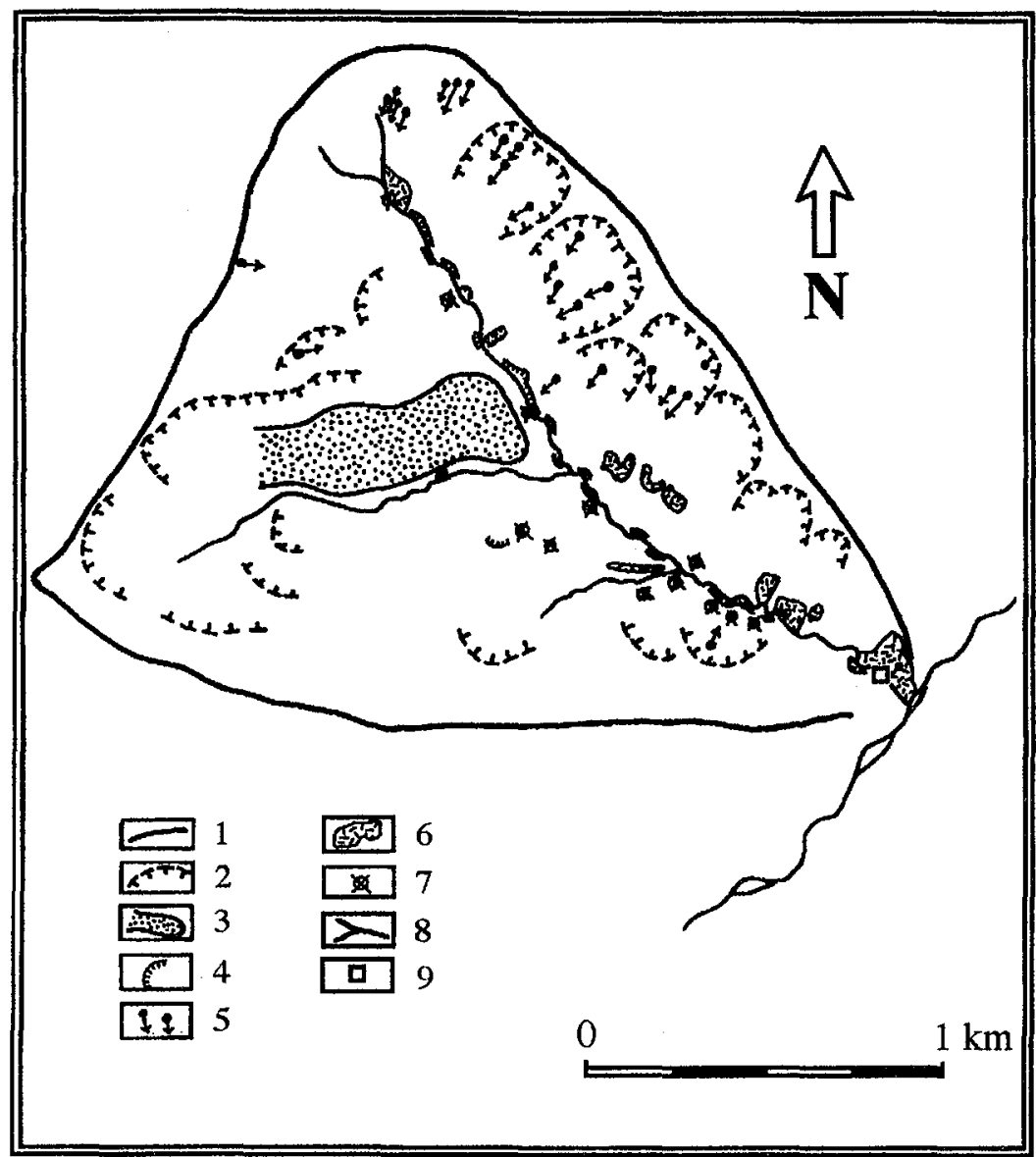

Figura 2. Esquema geomorfológico de la cuenca de Arnás. 1: Divisoria principal.

2: Cicatrices supuestas de antiguos deslizamientos profundos. 3: Lenguas de destizamiento. 4: Cicatrices de deslizamiento. 5: Coladas de piedras. 6: Áreas afectadas por erosión difusa severa. 7: Areas frecuentemente saturadas. 8: Red fluvial. 9: Localización del flume y de la estación meteorológica.

\section{Objetivos}

El principal objetivo de los trabajos llevados a cabo en Arnás y San Salvador es el estudio del comportamiento hidrológico y geomorfológico de dos cuencas con diferente historia de usos del suelo y de cubierta vegetal. Se trata, por lo tanto, de comprobar los efectos de los usos del suelo sobre la generación de escorrentía y la capacidad de transporte de sedimento, con la perspectiva global que da la escala cuenca y disponiendo además de información continua sobre los parámetros más importantes.

El equipamiento instalado en ambas cuencas permite disponer de información sobre las entradas (en forma de precipitaciones) y las salidas (en forma de caudal y distintos tipos de sedimento). Existen, evidentemente, notables relaciones entre entradas y salidas, pero no son tan marcadas como cabría esperar, de manera que el grado de incertidumbre de la respuesta hidrológica y geomorfológica es extraordinariamente alto. El papel de estas cuencas experimentales es contribuir a despejar esa incertidumbre mediante el estudio de una serie de procesos internos que tienen en cuenta las características de cada una de las cuencas (topografia, permeabilidad 
de los suelos, cubierta vegetal, sedimento disponible). De ahí la existencia de otros objetivos secundarios:

- Variabilidad espacial y temporal de la humedad del suelo.

- Variabilidad espacial y temporal de las áreas de contribución parcial.

- Mecanismos de generación de escorrentía.

- Identificación de las fuentes de sedimento.

- Importancia estacional de la interceptación por parte del matorral y del bosque.

\section{Equipamiento y métodos}

A la salida de ambas cuencas se ha construido un flume y se ha instalado el siguiente equipamiento:

- Sensor de ultrasonidos para la medición continua de la altura del agua (Lundhal DCU-7110), si bien en la cuenca de Arnás se montó inicialmente un sensor de presión con ocasionales fallos de funcionamiento, especialmente en periodos de aguas bajas.

- Turbidímetro (LYX 800 PT1) para la medición continua de la turbidez del agua, posibilitando, tras calibrado, el cálculo de la concentración de sedimento en suspensión.

- Conductivímetro para la medición continua de la conductividad eléctrica del agua. De momento sólo se ha instalado en la cuenca de Arnás, con importantes problemas, ya resueltos, debido a la sedimentación de carbonatos en el electrodo.

- Tomamuestras automático de agua (ISCO 3700) con capacidad para 24 botellas, programado para dispararse en diferentes momentos durante la fase ascendente del hidrograma en cuanto el nivel del agua alcanza $22 \mathrm{~cm}\left(4071 \mathrm{~s}^{-1}\right)$.

Estos sensores están conectados a data loggers que toman información cada 5 segundos, registrando la media cada 5 minutos. Los datos son descargados periódicamente mediante un ordenador portátil.

También se dispone de una trampa de sedimento localizada a la entrada de cada una de las estaciones de aforo, con una capacidad aproximada de $2.000 \mathrm{~kg}$, preparada para atrapar el sedimento que circula como carga de fondo.

Además, junto a cada aforo se ha instalado una estación meteorológica equipada con termómetro, higrómetro, anemómetro, piranómetro y pluviómetro, conectada a un data logger.

De esta forma, las dos cuencas están preparadas para la elaboración de balances hídricos y de sedimento (en solución, suspensión y de fondo) a distintas escalas temporales.

La cuenca de Arnás funciona de manera relativamente adecuada desde 1996, con una prolongada ausencia de datos en 1998. La cuenca de San Salvador no inició su funcionamiento de forma regular hasta principios de 1999, si bien se mantienen problemas en la obtención de información sobre trạnsporte de sedimento en suspensión. 
En diferentes condiciones topográficas de la cuenca de Arnás (solana/umbría, ladera cóncava/recta/convexa, parte alta/parte baja de la ladera) se instalaron 25 sondas de $30 \mathrm{~cm}$ de longitud para la medición de la humedad del suelo mediante TDR. Los datos se descargaron cada 7-14 días durante las estaciones húmedas y una vez al mes en verano durante los años 1996 y 1997.

Hasta el momento los trabajos realizados en las cuencas de Arnás y San Salvador se han centrado en el estudio del comportamiento hidrológico y sedimentológico de eventos de distinta magnitud. Para ello se han seleccionado las crecidas registradas en cada una de las cuencas y se ha elaborado una base de datos con información sobre:

i) diferentes rasgos de la precipitación: volumen total e intensidad en 5, 30 y 60 minutos, lluvia antecedente;

ii) características hidrológicas de la crecida: duración de la avenida, pico de caudal, caudal específico, pico de escorrentía directa (descontando el flujo de base), tiempo de respuesta (diferencia en minutos entre el inicio de la precipitación y el pico de crecida) y coeficiente de escorrentía;

iii) características del transporte de sedimento: pico de concentración del sedimento en suspensión, exportación total de sedimento en suspensión y tiempo de respuesta del sedimento.

Con la información disponible se han estimado balances de agua y sedimento para cada evento hidrológico, se han elaborado matrices de correlación y se han aplicado Análisis de Varianza (Anova). Diversas regresiones múltiples paso a paso han permitido identificar los factores que se muestran más directamente implicados en la variabilidad de los picos de crecida y en el transporte de sedimento en suspensión.

Las muestras de agua obtenidas con los tomamuestras automáticos se han analizado en el Laboratorio del Instituto Pirenaico de Ecología (Zaragoza) y permiten conocer la evolución de diferentes solutos durante la crecida.

\section{Principales resultados obtenidos}

\subsection{La frecuencia e intensidad de crecidas en Arnás y San Salvador}

La cuenca de Arnás presenta una frecuencia de crecidas más de tres veces superior a la de San Salvador, sin que existan diferencias significativas en cuanto al volumen o intensidad de precipitaciones. En las dos cuencas la crecida más intensa registrada se produjo en la misma fecha ( 9 de mayo de 2000), con una precipitación de $36,6 \mathrm{~mm}$ en Arnás y 51,2 $\mathrm{mm}$ en San Salvador. El pico de caudal en Arnás fue de $3.8741 \mathrm{~s}^{-1}\left(1.3601 \mathrm{~s}^{-1} \mathrm{~km}^{-2}\right)$ y en San Salvador $1.0861 \mathrm{~s}^{-1}\left(1.2901 \mathrm{~s}^{-1} \mathrm{~km}^{-2}\right)$.

La Fig. 3 muestra los hidrogramas y hietogramas correspondientes al periodo que va del 1 de octubre de 1999 al 30 de septiembre de 2000 en las cuencas de Arnás y San Salvador. La comparación de las dos cuencas permite comprobar que hay una clara coincidencia en los periodos de precipitación y en las fluctuaciones de caudal, si bien hay notables diferencias de comportamiento: 

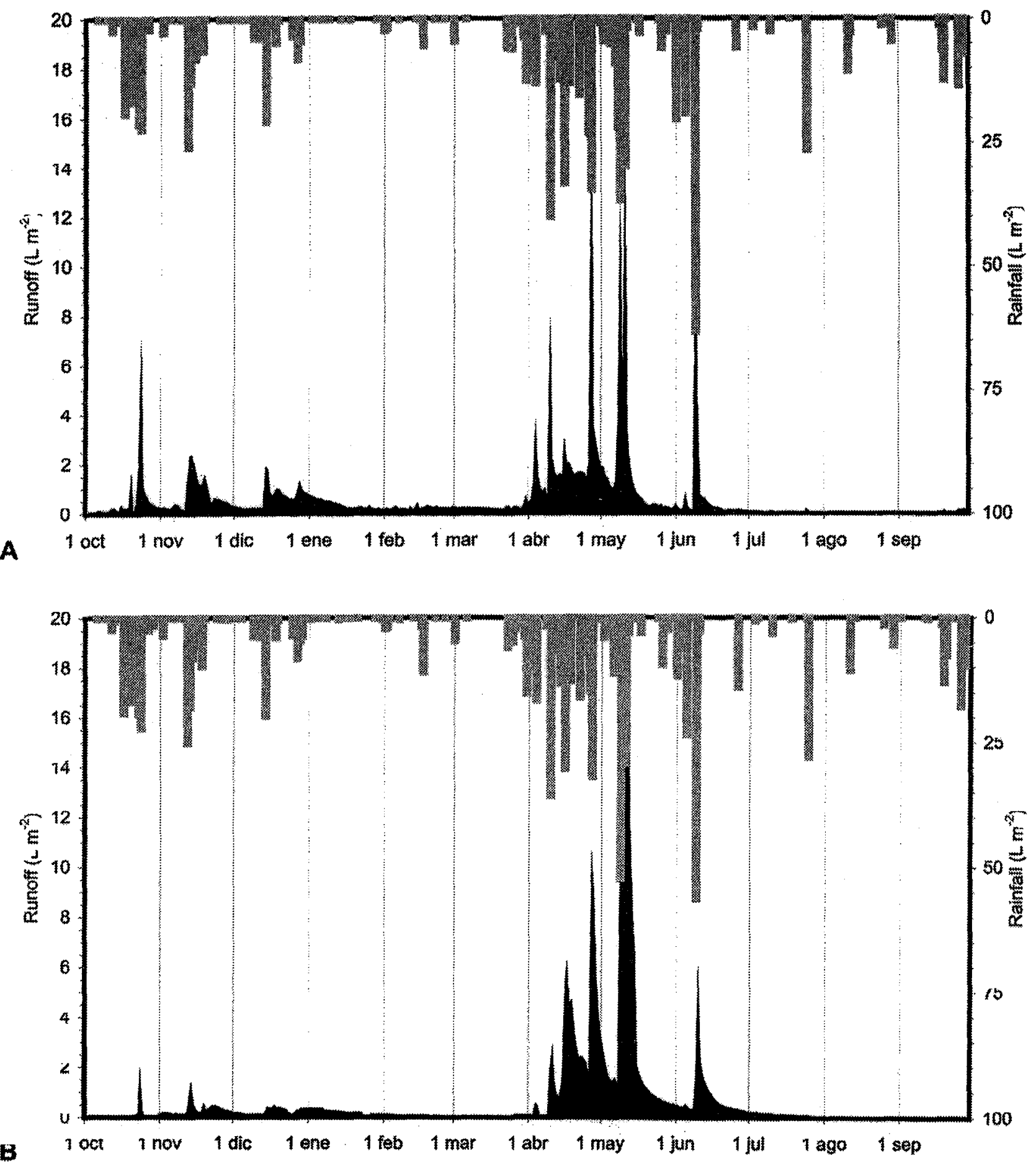

Figura 3. Precipitación y escorrentía en las cuencas de Arnás (A) y San Salvador (B) durante el periodo comprendido entre el 1 de octubre de 1999 y el 30 de septiembre de 2000.

- Durante las primeras lluvias de otoño no hay respuesta en la cuenca de San Salvador, mientras que Arnás presenta pequeños picos. Una lluvia de finales de octubre es capaz de producir un pico relevante en Arnás y sólo un ligero incremento de caudal en San Salvador. El mismo patrón se mantiene en diciembre y enero.

- En primavera del 2000 tiene lugar un prolongado e intenso periodo de precipitaciones, con fuertes incrementos de caudal en ambas cuencas, aunque más elevados en Arnás que en San Salvador. 
En la Fig. 4 se relacionan los valores medios de la precipitación y su correspondiente pico de crecida (en $1 \mathrm{~s}^{-1} \mathrm{ha}^{-1}$ ) en todas las crecidas registradas en Arnás y San Salvador durante el periodo en que se dispone de datos en ambas cuencas. En el caso de Arnás se distingue además entre crecidas que sólo han ocurrido en esta cuenca (Arnás 1) y aquellas que han coincidido con crecidas en la cuenca de San Salvador (Arnás 2). Las crecidas exclusivas de la cuenca de Arnás se caracterizan por valores bajos de precipitación y escorrentía. En el caso de las crecidas coincidentes, San Salvador se caracteriza por valores altos de precipitación y valores bajos de pico de crecida específico en comparación con Arnás (valores más bajos de precipitación y muy altos de pico de crecida específico).

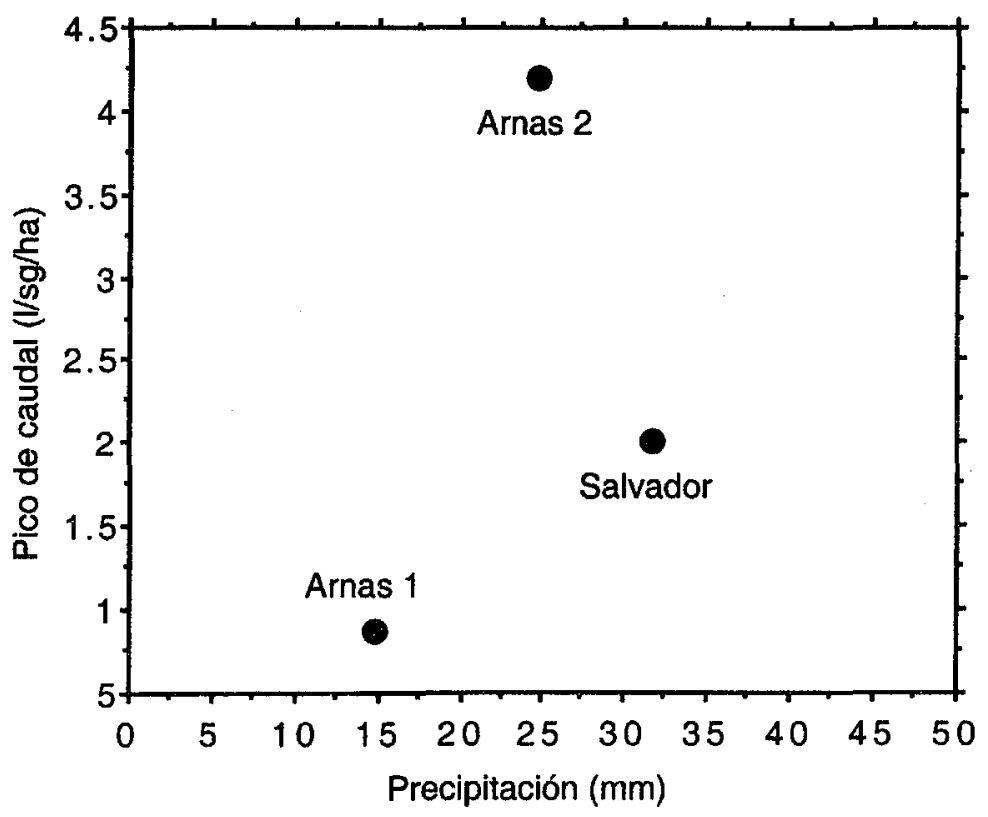

Figura 4. Valores medios de precipitación y picos de caudal en las cuencas de Arnás y San Salvador. Arnás 1: Crecidas sin réplica en San Salvador. Arnás 2: Crecidas con réplica en San Salvador.

La Fig. 5 muestra la correlación entre la precipitación y el pico de crecida en Arnás y San Salvador, con un mejor ajuste en esta última $\left(r^{2}=0.50 ; p=0.05\right)$. Aunque las nubes de puntos indican cierta dispersión, se observa que la recta correspondiente a Arnás se encuentra claramente por encima de la de San Salvador. El hecho de que tiendan a aproximarse conforme se elevan los valores de precipitación sugiere que en periodos húmedos los coeficientes de escorrentía tienden a equipararse debido a la menor incidencia de la interceptación en la cuenca forestal.

\subsection{Rasgos principales de las crecidas}

Si se consideran sólo las crecidas registradas en la cuenca de Arnás, la correlación entre precipitación y pico de crecida es muy baja $\left(r^{2}=0.33 ; p=<0.001\right)$ (Fig. 6). Este resultado tan pobre significa que, aunque la precipitación total de un evento lluvioso es un factor importante para explicar los picos de crecida, la intensidad de la respuesta hidrológica está también controlada por otros factores. De hecho, pueden registrarse picos de caudal de variado nivel casi con independencia del volumen total de precipitación. 


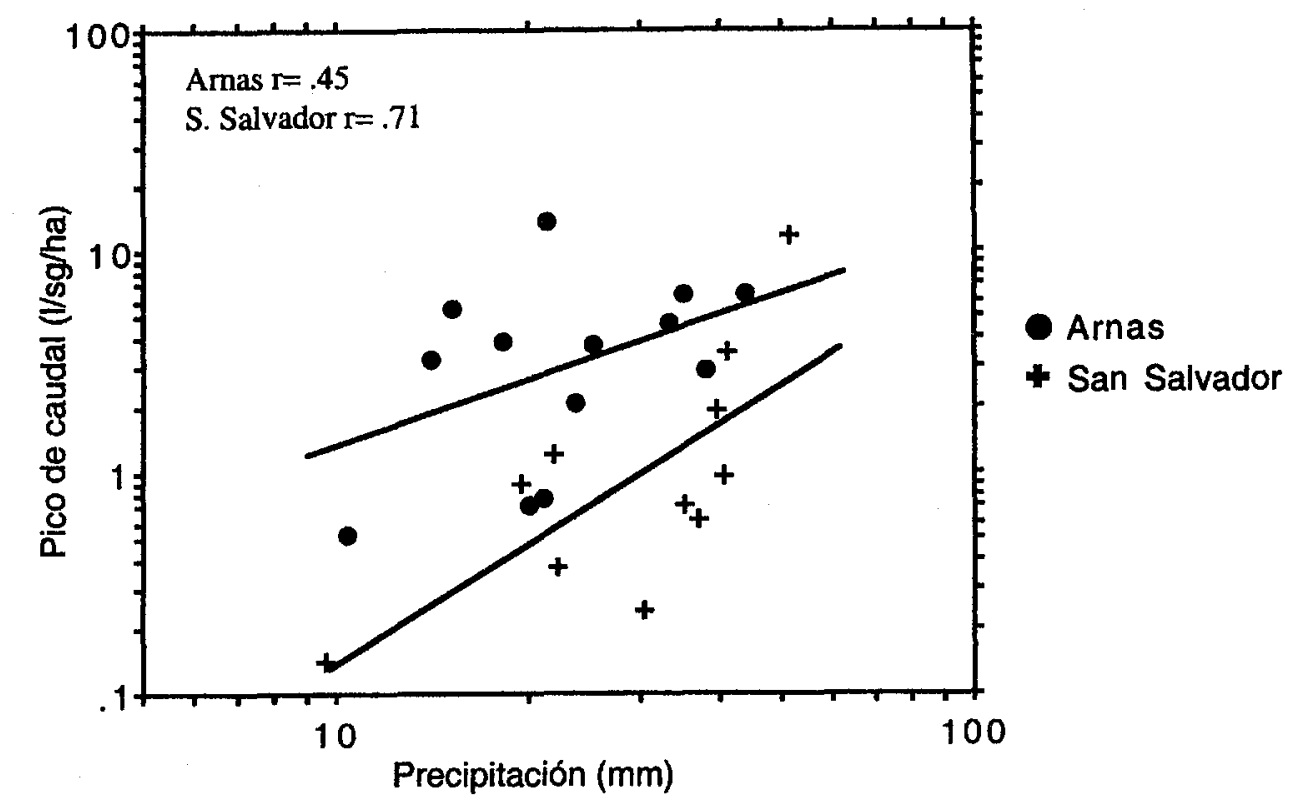

Figura 5. Relaciones entre precipitación y pico de crecida en las cuencas de Arnás y San Salvador. Se consideran sólo los eventos coincidentes en ambas cuencas.

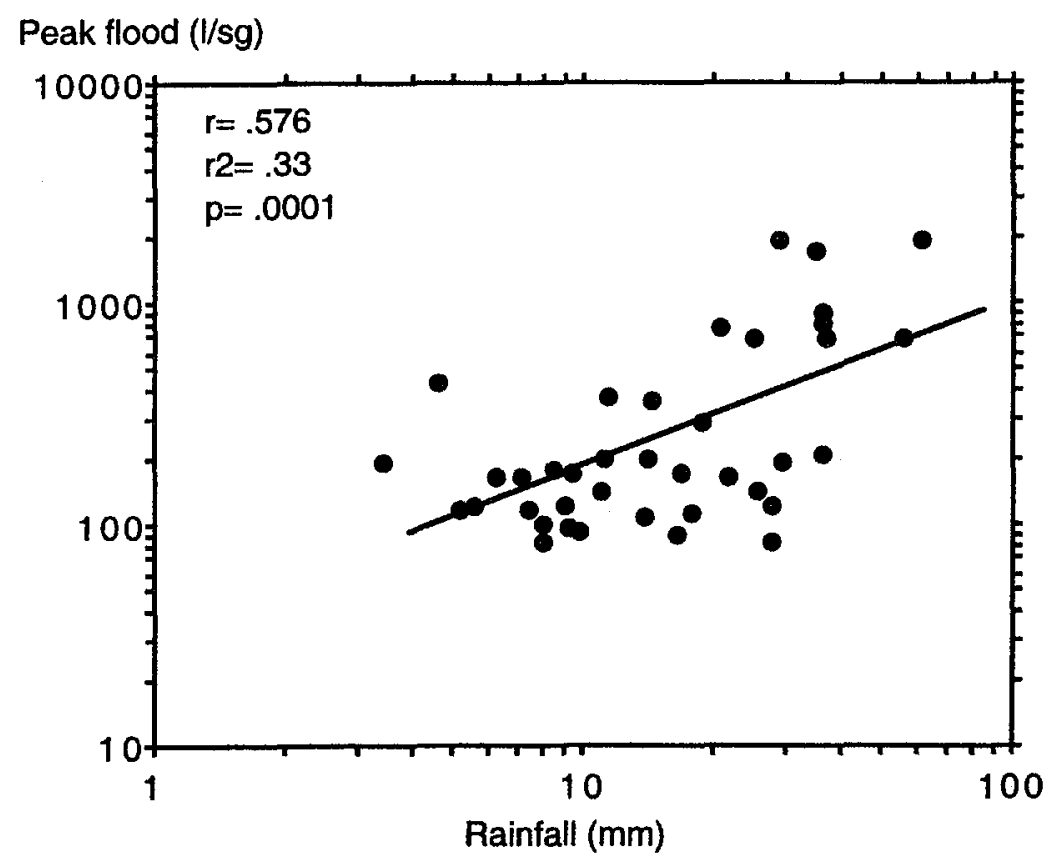

Figura 6. Relaciones entre la precipitación y el pico de caudal en las crecidas de la cuenca de Arnás.

La relación mejora sensiblemente si se consideran por separado las crecidas de estación fría y de estación cálida. En el primer caso la precipitación causante de cada crecida produce un coeficiente de determinación $\left(\mathrm{r}^{2}\right)$ de $0.41(\mathrm{p}=<0.001)$ (Arnáez et al., 1999). En el caso de las crecidas de estación cálida, el coeficiente de determinación es de sólo $0.20(\mathrm{p}=0.092)$ para la precipitación total.

Es evidente que el estado de humedad del suelo es un factor de gran relevancia para explicar la variabilidad de la respuesta hidrológica frente a precipitaciones dife- 
rentes. La Fig. 7 muestra la evolución de la humedad del suelo en la cuenca de Arnás, tomando como ejemplo dos puntos de la solana y de la umbría. Los valores más elevados se concentran durante la estación fría y principios de la cálida, mientras que al final de esta última se registran las cifras más bajas (García Ruiz et al., 1999).

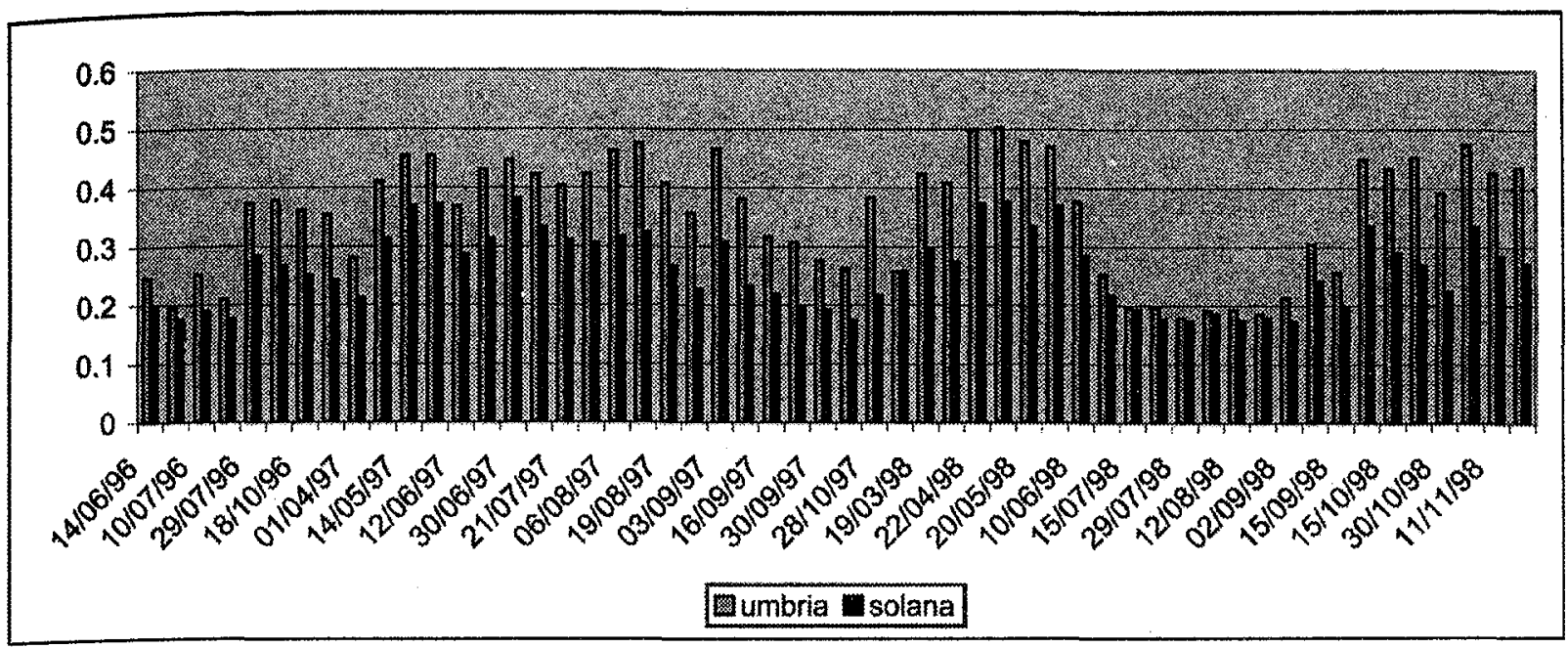

Figura 7. Evolución de la bumedad del suelo en la cuenca de Arnás en 1996 y 1997, distinguiendo entre la ladera solana y la umbría.

La existencia de una baja reserva de humedad en el suelo explica el que la respuesta hidrológica durante la estación cálida sea muy irregular, pues la mayor parte de la precipitación se consume en recargar el contenido de humedad del suelo. La realidad es que las primeras lluvias tras el verano apenas generan escorrentía y lo mismo sucede con algunas tormentas de verano, que pueden ser totalmente asimiladas por los suelos de la cuenca sin que se registre cambio alguno en el hidrograma (ver también Llorens et al., 1997). En cambio, durante la estación fría la respuesta dependerá más del volumen e intensidad de la precipitación, al destinarse sólo una pequeña proporción de la precipitación a generar áreas saturadas. De hecho, los estudios realizados hasta ahora demuestran que la precipitación total registrada durante los días anteriores a una crecida se correlaciona bien con los residuales de la regresión entre precipitación y pico de crecida (Arnáez et al., 1999). Es más, se ha podido comprobar que esa correlación se va debilitando a medida que aumenta la escala temporal considerada; es decir, la lluvia caída en los 3 días anteriores tiene mayor influencia que la caída en los 7 días anteriores y mucha mayor que la caída 15 y 21 días antes.

Varios hechos sugieren que la generación de escorrentía en la cuenca se relaciona más con la saturación del suelo que con el exceso de intensidad de la precipitación (modelo hortoniano) (Beguería et al., en prensa). Tanto la importancia de la humedad antecedente como el que las precipitaciones más intensas no produzcan necesariamente caudales mayores reflejan que la escorrentía de tormenta requiere una progresiva ampliación de las áreas saturadas o de contribución parcial. La ausencia de una respuesta hidrológica acusada durante las tormentas de verano o durante las primeras lluvias de otoño (incluso aunque sean intensas) es 
una prueba más de que la cuenca reacciona mejor frente a los mecanismos de saturación que a los de intensidad de la precipitación. De todas formas, si se comparan las crecidas de la cuenca de Arnás con las de la cuenca de San Salvador, se concluye que en esta última la variabilidad del pico de crecida se explica aún más que en la cuenca de Arnás por el volumen total de precipitación, sin que influya su intensidad, como también sucede con las cuencas experimentales de Vallcebre (Gallart et al., 1994).

\subsection{El transporte de sedimento}

La Fig. 8 muestra dos ejemplos de crecidas de acuerdo con las relaciones que se establecen entre caudal (hidrograma) y concentración de sedimento en suspensión (sedigrama).

i) El pico de transporte de sedimento en suspensión precede ligeramente al pico de crecida. Se trata de un fenómeno típico de lluvias cortas y no necesariamente intensas, en cuencas pequeñas con una rápida respuesta del transporte de sedimento. Las curvas de histéresis resultantes son positivas (Fig. 9).
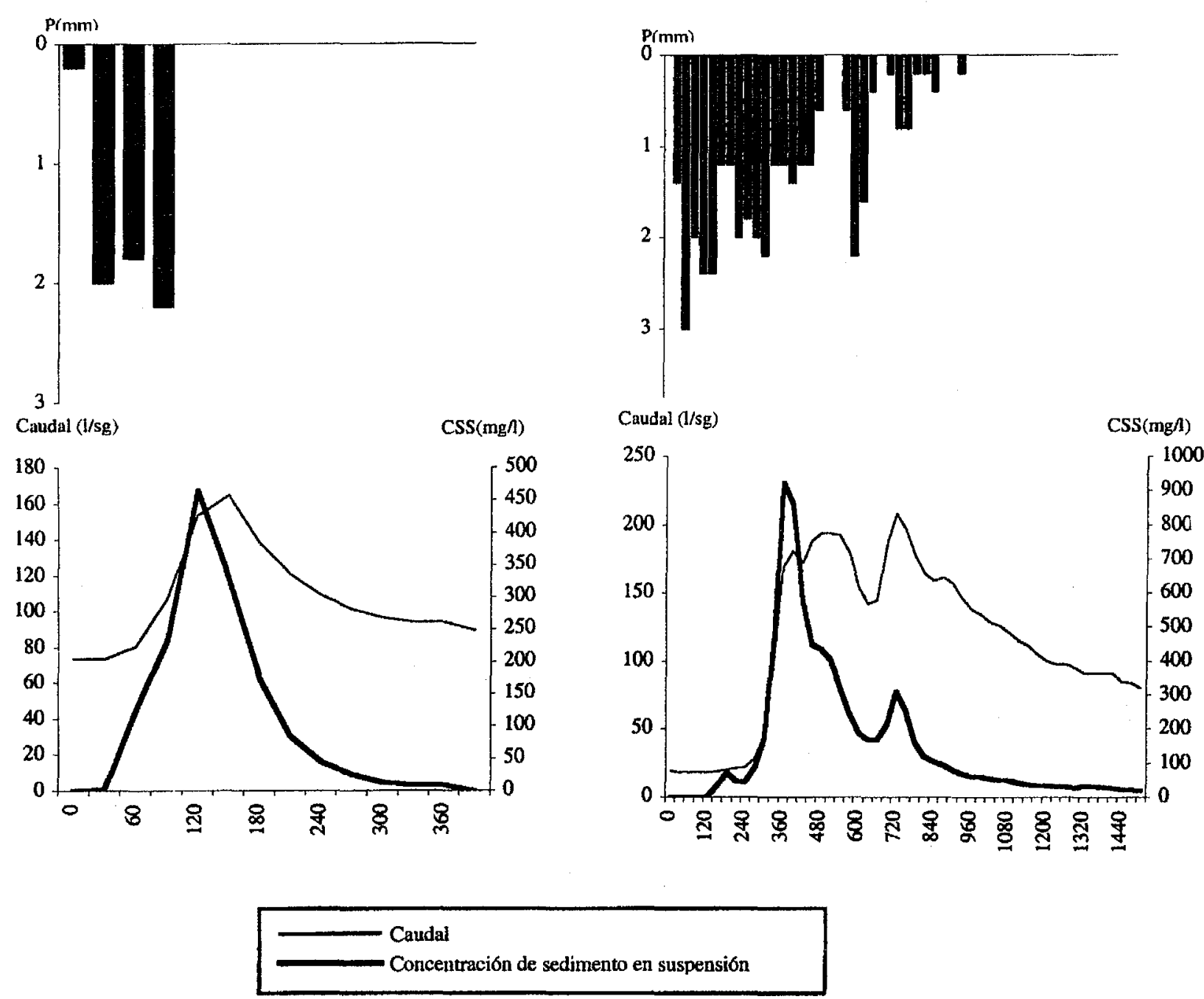

Figura 8. Modelos de relaciones entre precipitación, caudal y concentración de sedimento en suspensión durante crecidas en la cuenca de Arnás. 


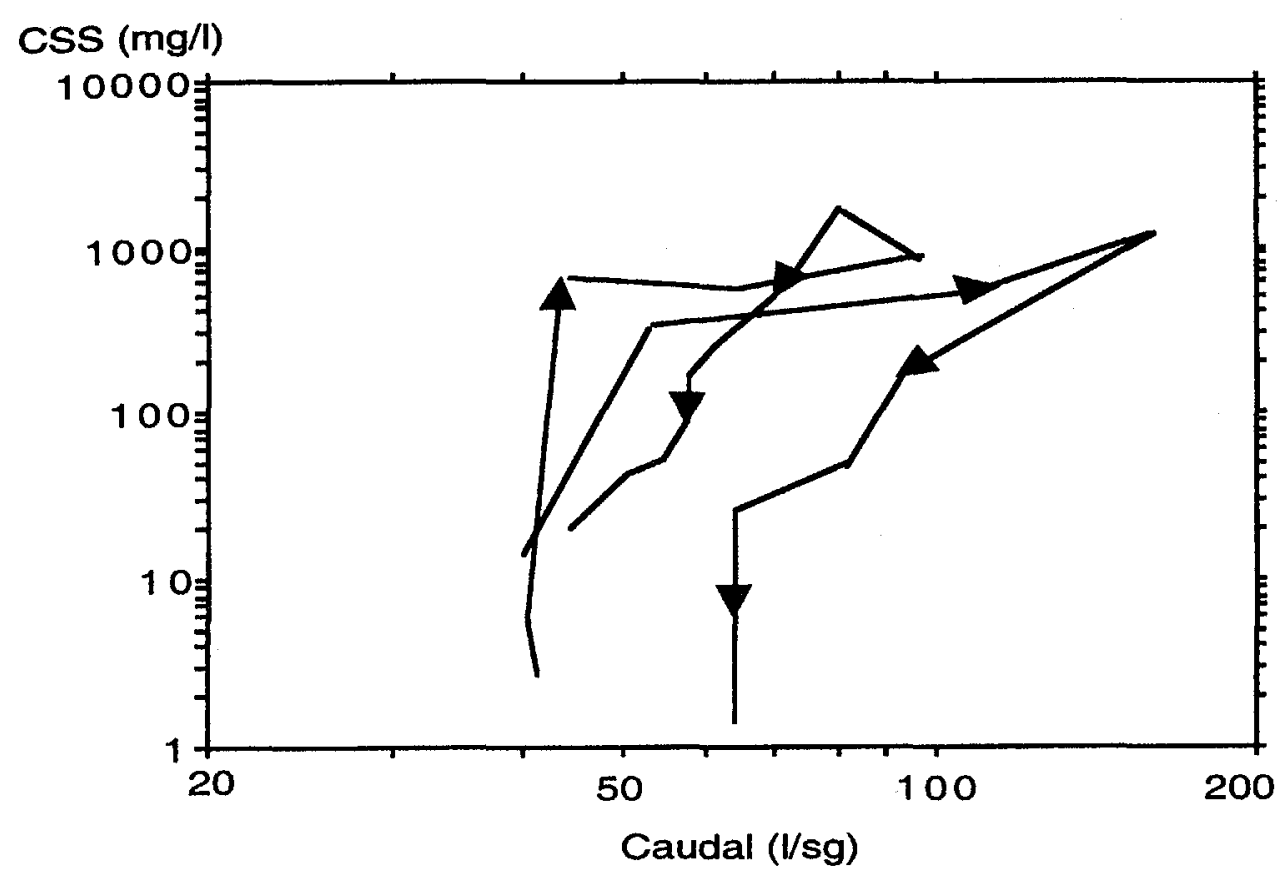

Figura 9. Curvas de bistéresis de dos crecidas consecutivas en la cuenca de Arnás.

ii) Durante crecidas de larga duración pueden producirse dos o más picos de caudal, coincidiendo con otros tantos periodos de precipitación más intensa. Lo que mejor caracteriza a este grupo de crecidas es que el segundo pico de concentración de sedimento es claramente inferior al primero. Este fenómeno podría atribuirse inicialmente a un efecto de agotamiento de sedimento en el cauce (Arnáez et al., 1998), pero esta interpretación no es muy aceptable dado que el material fino está disponible de forma casi ilimitada tanto en el cauce como en los taludes directamente afectados por la crecida. Una posibilidad es que se deba a un efecto de dilución (Lorente et al., 2000), ya que la cuenca está más húmeda durante el segundo pico de crecida, y las áreas más vegetadas pueden suministrar agua limpia (Gallart et al., 1998). También puede apuntarse que el flujo de base debe ser superior en el segundo pico, lo que hace que el sedimento incorporado por este último reduzca su concentración; sin embargo, este fenómeno no ha podido ser demostrado hasta el momento (Beguería et al., en prensa). La Fig. 9 compara las curvas de histéresis de dos crecidas correspondientes al día 18.05.1996 y separadas entre sí por tan sólo 9 horas. La segunda de las avenidas registra valores similares o incluso inferiores de concentración de sedimento en suspensión con un caudal notablemente superior.

La Fig. 10 muestra que la relación entre el pico de crecida y el pico de concentración de sedimento en suspensión es bastante pobre. Si sólo se tienen en cuenta las crecidas de la estación fría (Fig. 11), entonces el coeficiente de correlación aumenta sensiblemente $(r=0.87 ; p=0.0001)$, mientras que en el caso de las crecidas de estación cálida el resultado carece de significación, debido a que incluso con caudales pequeños es posible una elevada concentración de sedimento en suspensión. Lorente et al. (2000) han demostrado que, en buena parte, la respuesta del 
sedimento está relacionada con el pico de tomenta (una vez restado el flujo de base) y la intensidad de la precipitación en 30 y 60 minutos, lo que sugiere la importancia del splash más que de otros procesos de arranque de materiales en el suelo.

Atnáez et al. (1998) realizaron un primer balance de sedimentos en la cuenca de Arnás. El sedimento en suspensión representaría el $55 \%$ del total, frente a un $40 \%$ de solutos y $5 \%$ de carga de fondo. Esta última, sin embargo, puede alcanzar mucha mayor importancia en eventos individuales de elevada intensidad, como señalaron González et al. (1997). Debe tenerse en cuenta, además, que los datos sobre carga de fondo presentan una gran incertidumbre, ya que una parte de la misma puede circular por encima de la trampa de sedimento después de que ésta se haya colmatado.

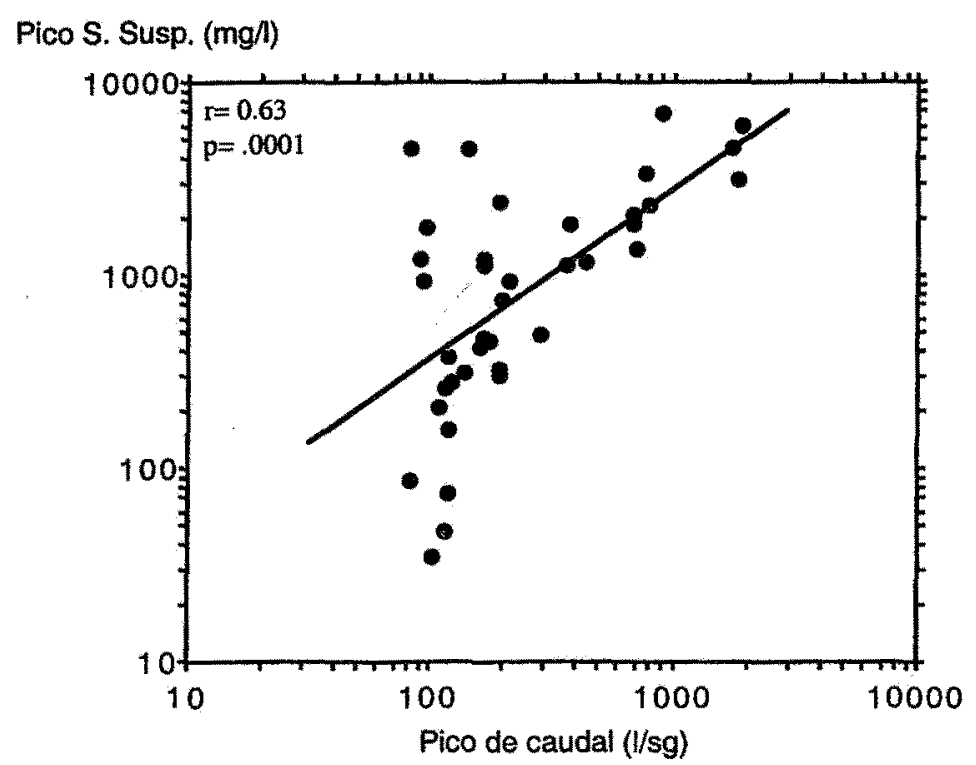

Figura 10. Relaciones entre el pico de caudal y el pico de concentración de sedimento en suspensión en crecidas de la cuenca de Arnás.

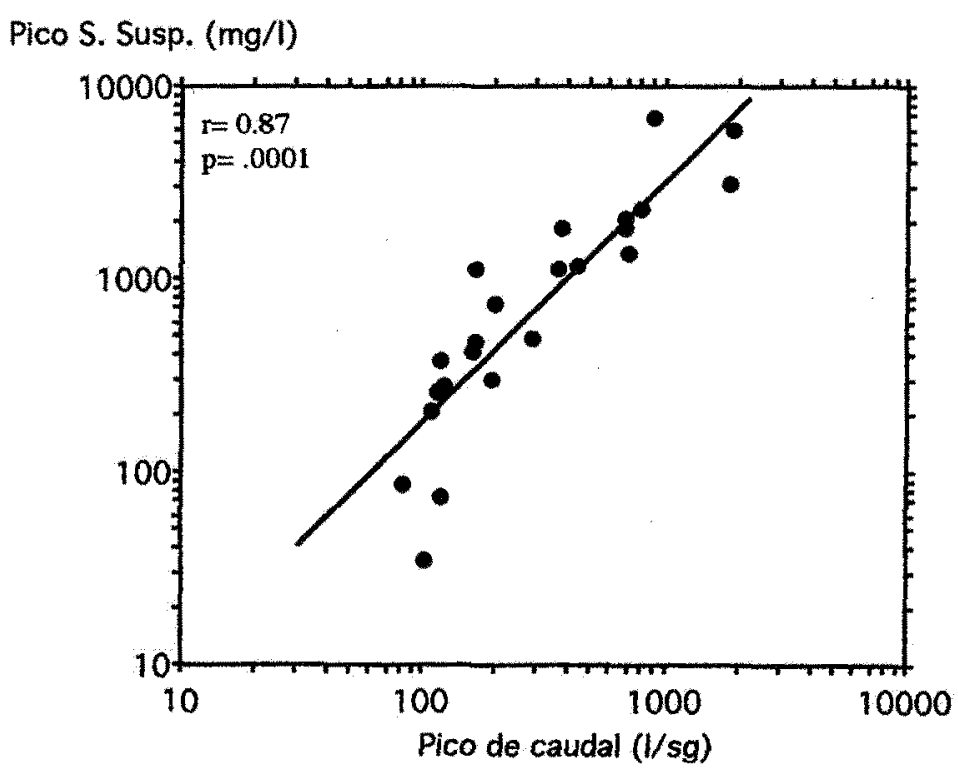

Figura 11. Relaciones entre el pico de caudal y el pico de concentración de sedimento en crecidas de la estación fría, cuenca de Arnás. 


\section{Conclusiones}

Los resultados obtenidos hasta ahora permiten, en primer lugar, comparar, aunque de forma muy elemental, el comportamiento hidrológico de dos cuencas con cubierta vegetal y usos de suelo muy diferentes y, en segundo lugar, identificar los rasgos más característicos de la generación de escorrentía y el transporte de sedimento en la cuenca de Arnás, que cuenta con una serie de datos más prolongada.

Cuando se comparan las dos cuencas, todos los resultados apuntan en la misma dirección: la cuenca forestal muestra un funcionamiento mucho más moderado que la cuenca de campos abandonados, a pesar de que esta última se halla densamente colonizada por distintas formaciones de matorral (Arnáez et al., en prensa). Ese funcionamiento se manifiesta en el hecho de que la cuenca de Arnás está afectada por un número mucho más elevado de crecidas, de forma que casi cualquier precipitación es capaz de generar un incremento significativo de caudal. En cambio, en la cuenca de San Salvador se necesita una precipitación superior a $20 \mathrm{~mm}$ para que pueda producirse una crecida. Además, en la mayor parte de las crecidas, el coeficiente de escorrentía de la cuenca de Arnás es claramente superior al de San Salvador, lo que sólo puede atribuirse a la interceptación de una parte de la lluvia por el bosque. De igual forma, el pico y volumen total de sedimento en suspensión transportado son mucho más elevados en Arnás que en San Salvador.

La relación entre precipitación y picos de crecida es muy baja en la cuenca de Arnás y sólo se obtienen resultados satisfactorios cuando se estudian por separado las crecidas de estación fría y las de estación cálida. Está claro que el estado previo de humedad del suelo ejerce una notable influencia sobre el patrón de respuesta hidrológica en cada evento pluviométrico. De ahí que las relaciones entre precipitación y caudal se ajusten mejor durante la estación fría, debido precisamente al efecto de las precipitaciones anteriores. Durante la estación cálida, el impacto de las precipitaciones registradas antes de cada crecida no es relevante debido a la intensa evapotranspiración y a la mayor separación temporal existente entre cada evento. Por otra parte, el hecho de que las precipitaciones antecedentes (o, lo que es lo mismo, el estado de humedad del suelo) expliquen buena parte de la variabilidad de los picos de crecida sugiere que la saturación es el mecanismo principal de generación de escorrentía dentro de la cuenca de Arnás, por encima de los procesos de tipo hortoniano (Beguería et al., en prensa).

El transporte de sedimento en suspensión presenta también una gran variabilidad, en parte relacionada con el pico de tormenta y, en parte con la intensidad de la precipitación (lo que permite intuir la importancia del splash en la liberación de partículas del suelo). El predominio de curvas de histéresis positivas durante las crecidas sugiere que las principales fuentes de sedimento en la cuenca de Arnás se localizan en el propio cauce, en sus taludes y áreas próximas afectadas por erosión difusa (González et al., 1997). Los estudios realizados sobre la distribución del Cesio $^{137}$ a cargo del Departamento de Edafología de la Estación Experimental de Aula Dei (García Ruiz et al., 1999) confirman que los suelos de la ladera solana han sido los más afectados por la erosión en las últimas décadas. Probablemente son también los que en condiciones de precipitaciones prolongadas pueden aportar cantidades 
importantes de sedimento, especialmente desde las laderas convexas próximas al cauce principal, coincidiendo con las áreas que han soportado condiciones más duras de explotación agrícola (García Ruiz et al., 1996b; Molinillo et al., 1997; García Ruiz, 1997). En todo caso, las histéresis positivas ponen de manifiesto lo que resulta cada vez más evidente en áreas de montaña abandonadas: la recolonización vegetal de los antiguos campos de cultivo tiende a provocar una contracción de la superficie ocupada por las fuentes más activas de sedimento (García Ruiz et al., 1996a; García Ruiz \& Valero Garcés, 1998). De hecho, los resultados obtenidos en parcelas experimentales (García Ruiz et al., 1995) confirman que el abandono de tierras de cultivo en laderas pendientes y la expansión de las formaciones densas de matorral representan un claro descenso en la exportación de sedimento en suspensión y de solutos. La inercia geomorfológica es, no obstante, muy grande: el predominio de los campos pendientes -no abancalados- en la cuenca de Arnás y la existencia de laderas afectadas por la agricultura itinerante hasta mediados del siglo XX (García Ruiz et al., 1996a), todavía contribuyen a explicar la gran importancia del sedimento en suspensión, en claro contraste con lo que sucede en cuencas dominadas por antiguas terrazas de cultivo (Llorens et al., 1997) y en cuencas forestales (Arnáez et al., en prensa).

\section{Agradecimientos}

Este trabajo se ha realizado con el apoyo de los siguientes proyectos de investigación: "Water resources management in a changing environment: The impact of sediment on sustainability" -WARMICE (ENV4-CT98-0789)-, financiado por la Comisión Europea, y "Estaciones permanentes para el estudio de procesos hidrológicos en ambientes mediterráneos" EPROHIDRO (HID98-1056-C02-01)- e "Identificación de fuentes de sedimento y áreas generadoras de escorrentía en relación con los cambios de uso del suelo" - HIDROESCALA (REN2000-1709-C04-01/GLO)-, financiados por la CICYT. Además, la monitorización de las cuencas se ha apoyado en parte en el Convenio CSIC/Ministerio de Agricultura (Dirección General de Conservación de la Naturaleza) a través de la RESEL.

\section{Bibliografía}

ARNÁEZ, J., BEGUERÍA, S., MARTÍ BONO, C., LORENTE, A. \& GARCÍA RUTZ, J.M. (en prensa): Avenidas y transporte de sedimento en cuencas con diferente cubierta vegetal. Estudio experimental en el Pirineo Central español. Actas de la VI Reunión de la Sociedad Española de Geomorfología, Madrid.

ARNÁEZ, J., GARCÍA RUIZ, J.M., MARTí BONO, C., BORDONABA, A., ERREA, M.P. \& WHITE, S., 1998. Avenidas y transporte de sedimentos en una pequeña cuenca de montaña media del Pirineo Central. En Investigaciones recientes de la Geomorfología española (A. Gómez Ortiz \& F. Salvador, eds.), Sociedad Española de Geomorfología, pp. 161-170, Granada.

ARNÁEZ, J., MARTÍ BONO, C., BEGUERÍA, S., LORENTE, A., ERREA, M.P. \& GARCÍA RUIZ, J.M., 1999. Factores en la generación de crecidas en una cuenca de campos abandonados, Pirineo Central español. Cuadernos de Investigación Geográfica, 25: 7-24. 
BEGUERÍA, S., MARTÍ BONO, C. \& ARNÁEZ, J. (en prensa): Mecanismos de generación de escorrentía directa a partir de datos hidrometeorológicos en una pequeña cuenca de media montaña, Pirineo Central.

GALLART, F., LLORENS, P. \& LATRON, J., 1994. Studying the role of old agricultural terraces on runoff generation in a small Mediterranean mountainous basin. Journal of Hydrology, 159: 291-303.

GALLART, F., LLORENS, P., LATRON, J., REGÜÉS, D. \& SALVANY, C., 1998. Las cuencas experimentales de Vallcebre (I): funcionamiento hidrológico. En Investigaciones recientes de la Geomorfología española (A. Gómez Ortiz \& F. Salvador, eds.), Sociedad Española de Geomorfología, pp. 187-192, Granada.

GARCÍA RUTZ, J.M., 1997. La agricultura tradicional de montaña y sus efectos sobre la dinámica hidromorfológica de laderas y cuencas. En Acción bumana y desertificación en ambientes mediterráneos (J.M. García Ruiz \& P. López García, eds.), Instituto Pirenaico de Ecología, pp. 119-144, Zaragoza.

GARCÍA RUIZ, J.M. \& GALLART, F., 1997. Las cuencas experimentales como base para el estudio de la erosión y la desertificación. En El paisaje mediterráneo a través del espacio y del tiempo. Implicaciones en la desertificación (J.J. Ibáñez, B.L. Valero \& C. Machado, eds.), Geoforma Ediciones, pp.: 221-238, Logroño.

GARCÍA RUIZ, J.M. \& VALERO GARCÉS, B., 1998. Historical geomorphic processes and human activities in the Central Spanish Pyrenees. Mountain Research and Development, 18 (4): 309-320.

GARCÍA RUIZ, J.M., LASANTA, T., ORTIGOSA, L., RUIZ FLAÑO, P., MARTÍ, C. \& GONZÁLEZ, C., 1995. Sediment yield under different land uses in the Spanish Pyrenees. Mountain Research and Development, 15 (3): 229-240.

GARCÍA RUIZ, J.M.. LASANTA, T., RUIZ FLAÑO, P., ORTIGOSA, L., WHITE, S., GONZÁlEZ, C. \& MARTÍ BONO, C., 1996a. Land-use changes and sustainable development in mountain areas: a case study in the Sapanish Pyrenees. Landscape Ecology, 11(5): 267-277.

GARCÍA RUIZ, J.M., LASANTA, T., GONZÁLEZ, C., MARTÍ, C., WHITE, S., ERREA, M.P. \& MAESTRO, M., 1996b. La agricultura marginal como fuente de sedimentos en el Pirineo central. Cadernos do Laboratorio Xeolóxico de Laxe, 21: 123-132.

GARCÍA RUIZ, J.M., WHITE, S., NAVAS, A. \& ERREA, M.P., 1999. CSIC/IPE contribution to the VAHMPIRE Project. En Validating bydrological models using process studies and internal data from research basins: Tools for assessing bydrological impacts of environmental change ( F. Gallart, S. White \& P. Llorens, eds.), European Commission, pp. 91-134, Barcelona.

GONZÁLEZ, C., GARCÍA RUIZ, J.M., MARTÍ, C., WHITE, S., ERREA, M.P. \& ARNÁEZ, J., 1997. Sediment sources in a small, abandoned farmland catchment, Central Spanish Pyrenees. Physics and Chemistry of the Earth, 22(3-4): 291-293.

LLORENS, P., QUERALT, I., PLANA, F. \& GALLART, F., 1997. Studying solute and particulate sediment transfer in a small Mediterranean mountainous catchment subject to land abandonment. Earth Surface Processes and Landforms, 22: 1027-1035.

LORENTE, A., MARTÍ BONO, C., BEGUERÍA, S., ARNÁEZ, J. \& GARCÍA RUIZ, J.M., 2000. La exportación de sedimento en suspensión en una cuenca de campos abandonados, Pirineo Central español. Cuaternario \& Geomorfología, 14 (1-2): 21-34. 
MOLINILLO, M., LASANTA, T. \& GARCÍA RUIZ, J.M., 1997. Managing mountainous degraded landscapes after farmland abandonment in the Central Spanish Pyrenees. Environmental Management, 21: 1-13.

WALLING, D.E., 1991. Drainage basin studies. En Field experiments and measurement programs in Geomorphology (O. Slaymaker, ed.), Balkema, pp. 17-59, Rotterdam.

Recibido en noviembre del 2000 Aceptado en enero del 2001 